\title{
Introduction to Section Two: MOOCs, Psychological Constructs, Communication Behaviors
}

\author{
Peter Shea, Editor-in-Chief, Online Learning \\ University at Albany, SUNY
}

This issue of Online Learning also contains four articles outside the theme of learning analytics. This section contains papers investigating MOOCs, a comparison of anxiety levels and the "imposter phenomenon" between online and classroom students, and a qualitative analysis of information behaviors among online students.

The first study is "Massive Open Online Courses (MOOCs): Participant Activity, Demographics, and Satisfaction” by Sara Shrader, Maryalice Wu, Dawn Owens, and Kathleen Santa Ana, of University of Illinois at Urbana-Champaign. In this paper the authors used clickstream and survey data to correlate demographic characteristics and levels of satisfaction with five separate clusters of participant activity that emerged from their analysis. They conclude that participants engage with MOOC courses in traditional and non-traditional ways, with some individuals choosing to participate in only one type of course activity, while others participate in multiple types of activity. Interestingly they found that noncompleters are neither unsuccessful nor inactive. While the traditional patterns of participation (perhaps resulting in "course completion") may be preferred, non-traditional pathways are also valuable given that they also result high levels of satisfaction for those who engage this way.

In the next study "Global Times Call for Global Measures: Investigating Automated Essay Scoring in Linguistically-Diverse MOOCs" by Erin Reilly, Kyle Williams, Rose Stafford, Stephanie Corliss, Janet Walkow and Donna Kidwell of the University of Texas at Austin the authors also investigate Massive Open Online Courses. Specifically this paper investigates the whether the use of Automated Essay Scoring (AES) with non-native speakers of English is justified. Given the scale of MOOCs the use of such technologies is a potentially attractive, time-saving approach; however their reliability in the MOOC context is not well understood. The authors conclude that although non-native English speakers performed lower on free form essay and short answer English assignments overall, the AES system itself may differentially disadvantage non-native English speaking students resulting in lower scores than assigned by human graders. Clearly additional work is needed in this area given the vast number of international students currently involved in MOOCs and the need to accommodate even greater demand going forward. 
Participation in graduate education can be a source of anxiety for many students. But do online environments present opportunities for reducing such anxiety and related negative psychological effects? These difficult questions are addressed in the next article, The Role of Social Influence in Anxiety and the Imposter Phenomenon by Christy Fraenza of Walden University. In this study the authors describe individual who suffer from the "imposter phenomenon" (IP) many of whom live their lives in constant fear of being discovered as less intelligent or competent than others believe them to be. The cycle of IP leaves the afflicted in a state of uncomfortable anxiety, especially in the face of imminent evaluations, such as those common to graduate education. One hypothesis guiding this study is that the source of anxiety triggering IP may be tied to social influence effects that are more salient in face-to-face settings. Does participation in online setting reduce the social influence that may result in anxiety tied to IP? Results indicated that graduate students in face-to-face settings had significantly higher IP scores than online graduate students. Results also indicated a significant, positive relationship between IP scores and anxiety scores. There are limitations to these findings of course, but the study raises very interesting issues for both classroom and online educators.

The final article in this section is Information Sharing, Community Development, and Deindividuation in the eLearning Domain by Nicole Cooke, of the University of Illinois at UrbanaChampaign. This study employs qualitative methods to provide a generative explanation of patterns of information interactions revealed in the written interactions of graduate students in an online learning community. Results provide a rich descriptive basis for understanding how students in an online Library and Information Science course create connections and build community over the span of a single term. The findings indicate that information behavior and sharing are not compartmentalized into strict cognitive and affective categories, but these domains complement one another to form a more comprehensive view of information seeking, utilization, and sharing, that contribute to the overall production of knowledge.

\section{References}

Cooke, Nicole A. (2016). Information sharing, community development, and deindividuation in the eLearning Domain. Online Learning, 20 (2), 244 - 260.

Fraenza, Christy B. (2016). The role of social influence in anxiety and the Imposter Phenomenon. Online Learning, 20 (2), 230 - 243.

Reilly, Erin D.; Williams, Kyle M.; Stafford, Rose E.; Corliss, Stephanie B.; Walkow, Janet C.; \& Donna K. Kidwell (2016). Global times call for global measures: Investigating automated essay scoring in linguistically-diverse MOOCs. Online Learning, 20 (2), 217 -229.

Shrader, Sara; Wu, Maryalice; Owens, Dawn \& Santa Ana, Kathleen (2016). Massive open online courses (MOOCs): Participant activity, demographics, and satisfaction. Online Learning, 20 (2), $199-216$. 